\title{
SHANNON ENTROPY AS A MEASURE OF UNCERTAINTY IN POSITIONS AND MOMENTA
}

\author{
Łukasz Rudnicki* \\ Center for Theoretical Physics, Polish Academy of Sciences \\ Aleja Lotników 32/46, PL-02-668 Warsaw, Poland \\ *Corresponding author e-mail: rudnicki@cft.edu.pl
}

\begin{abstract}
This paper is prepared as a contribution to the proceedings after the 12th ICSSUR/Feynfest Conference held in Foz do Iguaçu (Brazil) from 2 to 6 May 2011. In the first part I briefly report the topic of entropic uncertainty relations for position and momentum variables. Then I investigate the discrete Shannon entropies related to the case of finite number of detectors set to measure probability distributions in position and momentum spaces. I derive an uncertainty relation for the sum of the Shannon entropies which generalizes previous approaches [Phys. Lett. 103 A, 253 (1984)] based on an infinite number of detectors (bins).
\end{abstract}

Keywords: Shannon entropy, entropic uncertainty relations, uncertainty of quantum measurements performed with finite accuracy and finite number of detectors

\section{Introduction}

Entropic uncertainty relations for position and momentum, or other canonically conjugated variables have been derived a long time ago. Initial investigations [1] were devoted to continuous Shannon entropies. Further generalizations took into account, in a spirit of Deutsch [2] and Maassen-Uffink [3] results, the accuracy of measuring devices [4-8] and impurity of a quantum state [9]. Recent 12th ICSSUR/Feynfest Conference showed that the topic of entropic uncertainty relations including experimental accuracies is important in the task of entanglement detection in quantum optics 10, 11].

This paper is organized as follows. In a further part of the present section I point out some aspects related to entropic uncertainty relations, following the rephrased Heisenberg sentence [8], the more information we have about the position, the less information we can acquire about the momentum and vice versa" and an observation made by Peres [12], , The uncertainty relation such as $\sigma_{x} \sigma_{p} \geq \hbar / 2$ is not a statement about the accuracy of our measuring instruments". It the second section I generalize an approach presented in [5] to the case of finite number of measuring devices (detectors). 


\subsection{Entropic uncertainty relations and continuous Shannon entropy}

I would like to start with the famous Bialynicki-Birula-Mycielski entropic uncertainty relation of the form [1]

$$
-\int_{-\infty}^{\infty} d x \rho(x) \ln \rho(x)-\int_{-\infty}^{\infty} d p \tilde{\rho}(p) \ln \tilde{\rho}(p) \geq 1+\ln \pi \hbar
$$

where $\rho(x)=|\psi(x)|^{2}$ and $\tilde{\rho}(p)=|\tilde{\psi}(p)|^{2}$ are probability distributions in position and momentum spaces respectively. Wave functions in both spaces are related to each other by the Fourier transform

$$
\tilde{\psi}(p)=\frac{1}{\sqrt{2 \pi \hbar}} \int_{-\infty}^{\infty} d x e^{-i p x / \hbar} \psi(x) .
$$

The introduction of [1] starts as follows:

,, The purpose of this paper is to derive a new stronger version of the Heisenberg uncertainty relation in wave mechanics. This new uncertainty relation has a simple interpretation in terms of information theory. It is also closely related to newly discovered logarithmic Sobolev inequalities."

Information theory enters to the inequality (1) by the notion of continuous Shannon entropies $S^{(x)}=$ $-\int_{-\infty}^{\infty} d x \rho(x) \ln \rho(x)$ and $S^{(p)}=-\int_{-\infty}^{\infty} d p \tilde{\rho}(p) \ln \tilde{\rho}(p)$. Connection with the logarithmic Sobolev inequality can be recognized with the help of the reversed logarithmic Sobolev inequality [13 which, for the probability distribution function $(\mathrm{PDF}) f(z)$ defined on $\Omega \subset \mathbb{R}$, reads

$$
\int_{\Omega} d z f(z) \ln f(z) \geq-\frac{1}{2} \ln \left[2 \pi e \sigma_{z}^{2}(\Omega)\right]
$$

The variance is defined as usual, $\sigma_{z}^{2}(\Omega)=\int_{\Omega} d z z^{2} f(z)-\left(\int_{\Omega} d z z f(z)\right)^{2}$. We shall use the inequality (3) independently for the position and momentum variables and obtain the stronger version of the Heisenberg uncertainty relation, announced in 1

$$
\sigma_{x} \sigma_{p} \geq \frac{\hbar}{2} \exp \left(S^{(x)}+S^{(p)}-1-\ln \pi \hbar\right) \geq \frac{\hbar}{2}
$$

\subsubsection{Continuous Shannon entropy as a measure of information}

It seems to be widely accepted that the continuous Shannon entropy is also a good measure of information since it is a relative of Shannon information entropy. However, this statement is not completely true. To prove that let me recall the definition of the Shannon entropy of a set of probabilities $\left\{P_{i}\right\}$

$$
H^{(P)}=-\sum_{i} P_{i} \ln P_{i}
$$

We have two important properties of the Shannon entropy (5):

1. Since the probabilities $P_{i}$ are dimensionless the Shannon entropy $H^{(P)}$ is also dimensionless.

2. From the property $0 \leq P_{i} \leq 1$ we know that $H^{(P)} \geq 0$. 


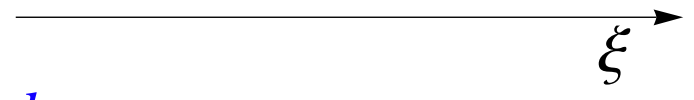

$b$

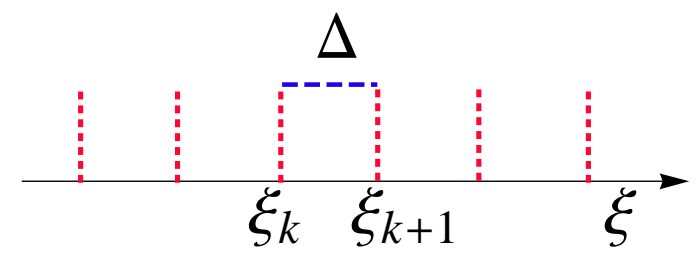

Figure 1: Partition of the real line into bins.

These two properties are essential for the information-like interpretation of the Shannon entropy because information can be neither negative nor expressed in any physical units. Unfortunately the continuous Shannon entropy does not possess these two properties. For instance the unit of the entropy $S^{(x)}$ (in SI units) is the logarithm of meter. This makes impossible to check if the continuous Shannon entropy is positive or negative. On the other hand if we introduce some length (momentum) scale in order to measure $S^{(x)}\left(S^{(p)}\right)$ then we still cannot fulfill the second property since $\rho(x)(\tilde{\rho}(p))$ can be greater than 1 and make the continuous entropy negative.

\subsection{Discrete Shannon entropies including experimental accuracies}

An idea that helps to overcome the difficulties which I have mentioned above (related to the continuous entropies) introduces a partition of the real line into bins of equal width (see Fig. 1).

As a result the continuous probability distribution $f(\xi)$ is replaced by a discrete distribution $P_{k}$, $(k \in \mathbb{Z})$

$$
P_{k}=\int_{\xi_{k}}^{\xi_{k+1}} d \xi f(\xi)
$$

The bins are of equal width, thus $\forall_{k} \xi_{k+1}-\xi_{k}=\Delta$. This idea was for the first time introduced by Partovi [4] who noticed that $\Delta$ shall be interpreted as a ,,resolution of the measuring device”.

\subsubsection{A remark about translational invariance}

The requirement that all bins have to have the same width $\Delta$ is a strong constraint on $\xi_{k}$. In fact $\xi_{k}$ must be of the form

$$
\xi_{k}=\xi_{0}+k \Delta
$$

so there is only one free parameter $\xi_{0}$ to be chosen. One might expect that different values of the parameter $\xi_{0}$ are equivalent to different possible choices of the central point $(\xi=0)$ of the used coordinate. In the first Partovi's paper [4] and later publications [5.6. 14] the easiest possible choice $\xi_{0}=0$ was made. In this choice the probability distributions and the Shannon entropies are $(\delta x$ and $\delta p$ are experimental accuracies for positions and momenta respectively):

$$
\mathcal{Q}_{k}=\int_{k \delta x}^{(k+1) \delta x} d x \rho(x), \quad \mathcal{P}_{l}=\int_{l \delta p}^{(l+1) \delta p} d p \tilde{\rho}(p),
$$




$$
H^{(x)}=-\sum_{k=-\infty}^{\infty} \mathcal{Q}_{k} \ln \mathcal{Q}_{k}, \quad H^{(p)}=-\sum_{l=-\infty}^{\infty} \mathcal{P}_{l} \ln \mathcal{P}_{l}
$$

In this moment I would like to point out that the entropies defined in (8, 9) are not correct measures of information (because of the choice $\xi_{0}=0$ ). In order to realize that one shall investigate the limit of large coarse graining (large experimental accuracies) $\delta x \rightarrow \infty$ or $\delta p \rightarrow \infty$. In these limits performed measurements tell us nothing about the quantum state - our information is 0 , thus, we shall expect $\lim _{\delta x \rightarrow \infty} H^{(x)}=\lim _{\delta p \rightarrow \infty} H^{(p)}=0$. But in fact, we have:

$$
\lim _{\delta x \rightarrow \infty} \mathcal{Q}_{k}=\left\{\begin{array}{ll}
\int_{0}^{\infty} d x \rho(x) & \text { for } k=0 \\
\int_{-\infty}^{0} d x \rho(x) & \text { for } k=-1
\end{array}, \quad \lim _{\delta p \rightarrow \infty} \mathcal{P}_{l}=\left\{\begin{array}{ll}
\int_{0}^{\infty} d p \tilde{\rho}(p) & \text { for } l=0 \\
\int_{-\infty}^{0} d p \tilde{\rho}(p) & \text { for } l=-1
\end{array} .\right.\right.
$$

As a result $\lim _{\delta x \rightarrow \infty} H^{(x)}\left(\lim _{\delta p \rightarrow \infty} H^{(p)}\right)$ have state-dependent values that vary between 0 if the state is localized in $\mathbb{R}_{+}$or $\mathbb{R}_{-}$(in positions or momenta) and $\ln 2$ if the state is symmetric.

In 8, 15 we captured this ambiguity and performed the redefinition of the probability distributions (8) in the following way:

$$
\mathcal{Q}_{k} \longmapsto q_{k}=\int_{(k-1 / 2) \delta x}^{(k+1 / 2) \delta x} d x \rho(x), \quad \mathcal{P}_{l} \longmapsto p_{l}=\int_{(l-1 / 2) \delta p}^{(l+1 / 2) \delta p} d p \tilde{\rho}(p) .
$$

It is equivalent to the choice $\xi_{0}=-\Delta / 2$ in the construction (7) and means that the center of the coordinate $\xi=0$ lays in the middle of the central bin. In the previous choice the center of the coordinate was the border point between two bins.

\subsubsection{Entropic uncertainty relations and recent results}

Similarly to (1) uncertainty relations for $H^{(x)}+H^{(p)}$ were found a long time 4, 5]. The stronger one [5] reads

$$
H^{(x)}+H^{(p)} \geq-\ln \left(\frac{\delta x \delta p}{e \pi \hbar}\right)=\mathcal{B} .
$$

Since the Shannon entropies (9) have been correctly defined (are positive and dimensionless) it is obvious that $H^{(x)}+H^{(p)} \geq 0$. Thus, for $\delta x \delta p \geq e \pi \hbar$ the relation 12 becomes trivially satisfied and in fact does not give an optimal lower bound (the bound is optimal, and saturated by a Gaussian distribution, only in the limit $\delta x \rightarrow 0$ and $\delta p \rightarrow 0$ ). Some attempts 14 to find a better lower bound failed completely (for details see [15] - the comment on [14]). Recently [16, 17] we derived the bound

$$
H^{(x)}+H^{(p)} \geq \max (\mathcal{B}, \mathcal{R}), \quad \mathcal{R}=-2 \ln \left[\sqrt{\frac{\delta x \delta p}{2 \pi \hbar}} R_{00}\left(\frac{\delta x \delta p}{4 \hbar}, 1\right)\right],
$$

which is always positive, but still not optimal (cf. Fig. 2). Function $R_{00}(\xi, \eta){ }^{1}$ is one of the radial prolate spheroidal wave functions of the first kind [18].

\footnotetext{
${ }^{1}$ in the Wolfram Mathematica's notation it reads SpheroidalS1 $[0,0, \xi, \eta]$.
} 


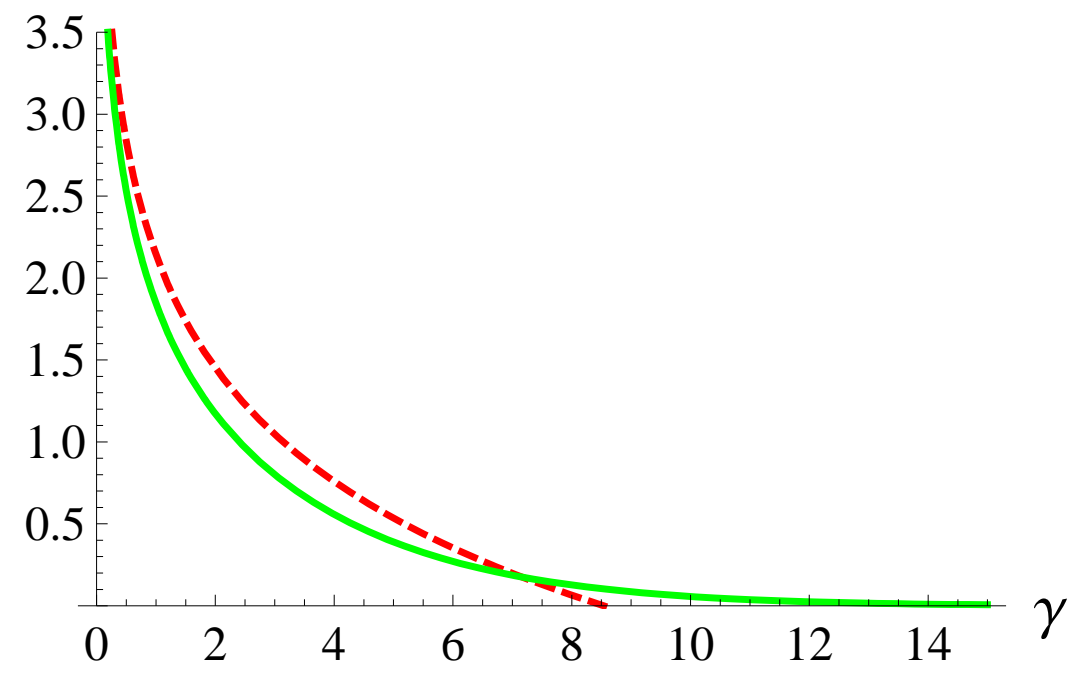

Figure 2: Comparison between two bounds in (13). Red/dashed curve represents $\mathcal{B}(\gamma)$ while green curve represents $\mathcal{R}(\gamma) ; \gamma=\delta x \delta p / \hbar$.

\section{Entropy based on a finite number of detectors}

In the definition (9) the summation over $k(l)$ goes from $-\infty$ to $+\infty$. That property in a language of an experiment means that there are infinitely many measuring devices (detectors) covering an infinite number of bins. Of course in realistic experiments only a finite number of bins can be covered, thus, we shall describe the measurement's result with the help of the finite Shannon entropies, defined as follows:

$$
H_{M}^{(x)}=-\sum_{k=-M}^{M} q_{k} \ln q_{k}, \quad H_{N}^{(p)}=-\sum_{l=-N}^{N} p_{l} \ln p_{l} .
$$

These definitions include $2 M+1$ detectors set to measure the probability distribution in positions and $2 N+1$ detectors for momenta. We have the limits $\lim _{M \rightarrow \infty} H_{M}^{(x)}=H^{(x)}$ and $\lim _{N \rightarrow \infty} H_{N}^{(p)}=H^{(p)}$. Since all terms in the sums in (9) are non-negative we know that $H_{M}^{(x)} \leq H^{(x)}$ and $H_{N}^{(p)} \leq H^{(p)}$. Thus, we cannot expect that the uncertainty relations 12 or 13 will be satisfied for $H_{M}^{(x)}+H_{N}^{(p)}$. Moreover we can always find many states localized far away from the detectors (with $|\langle x\rangle| \gg(M+1 / 2) \delta x$ and $|\langle p\rangle| \gg(N+1 / 2) \delta p)$, for example

$$
\psi(x)=\left(\frac{1}{\pi \sigma^{2}}\right)^{1 / 4} e^{i p_{0}\left(x-x_{0} / 2\right) / \hbar} \exp \left[-\frac{\left(x-x_{0}\right)^{2}}{2 \sigma^{2}}\right], \Longrightarrow \quad \rho(x)=\frac{1}{\sqrt{\pi} \sigma} \exp \left[-\frac{\left(x-x_{0}\right)^{2}}{\sigma^{2}}\right]
$$

with $x_{0}=\langle x\rangle$ and $p_{0}=\langle p\rangle$. This inconvenience is directly related to the translational invariance which was broken when we restricted the number of used bins. Of course an experimentalist knows where the measured state is and can properly choose the coordinate to assure that $\langle x\rangle=0=\langle p\rangle$.

Looking at Fig. 2 we can realize that if $\delta x \delta p / \hbar \lesssim 7.167$ then the previous lower bound $(12)$ dominates. I would like to work in this regime and, although the finite number of bins makes us unable to find a state independent lower bound for $H_{M}^{(x)}+H_{N}^{(p)}$, I would like to find a state-dependent correction to the 
formula (12). I shall start in the same way as was done in [5] and use the integral Jensen inequality (I will do the calculations only for the position variable since in the momentum case everything shall go the same way)

$$
\begin{aligned}
H_{M}^{(x)} \geq & -\int_{-(M+1 / 2) \delta x}^{(M+1 / 2) \delta x} d x \rho(x) \ln [\rho(x) \delta x] \\
& =B_{x}+q_{\infty} \ln (\delta x)+\left(\int_{-\infty}^{-(M+1 / 2) \delta x}+\int_{(M+1 / 2) \delta x}^{\infty}\right) d x \rho(x) \ln \rho(x),
\end{aligned}
$$

where I have introduced the following notation:

$$
B_{x}=-\ln (\delta x)+S^{(x)} \quad q_{\infty}=\left(\int_{-\infty}^{-(M+1 / 2) \delta x}+\int_{(M+1 / 2) \delta x}^{\infty}\right) d x \rho(x) .
$$

The Shannon entropy, because of the logarithmic function, collects information from all moments of the probability distribution. A convenient method to reduce the state-dependent input can be based on the reversed logarithmic Sobolev inequality (3). We shall choose $f(z)=\rho(z) / q_{\infty}$ and $\Omega_{M}=$ ]$-\infty,-(M+1 / 2) \delta x] \cup[(M+1 / 2) \delta x, \infty[$ and obtain

$$
\left(\int_{-\infty}^{-(M+1 / 2) \delta x}+\int_{(M+1 / 2) \delta x}^{\infty}\right) d x \rho(x) \ln \rho(x) \geq-\frac{q_{\infty}}{2} \ln \left(\frac{2 \pi e}{q_{\infty}^{2}} \sigma_{x}^{2}\left(\Omega_{M}\right)\right) .
$$

Since we expect that the state fulfills $\langle x\rangle \approx 0 \approx\langle p\rangle$ we can, without any significant loss, simplify the right hand side of 18

$$
\sigma_{x}^{2}\left(\Omega_{M}\right) \leq \frac{\left\langle x^{2}\right\rangle_{M}}{q_{\infty}}, \quad\left\langle x^{2}\right\rangle_{M}=\left(\int_{-\infty}^{-(M+1 / 2) \delta x}+\int_{(M+1 / 2) \delta x}^{\infty}\right) d x x^{2} \rho(x) .
$$

After this step we obtain the inequality

$$
H_{M}^{(x)} \geq B_{x}+R\left(q_{\infty},\left\langle x^{2}\right\rangle_{M}\right), \quad R(\eta, \Lambda)=\frac{\eta}{2} \ln \left(\frac{(\delta x)^{2} \eta^{3}}{2 \pi e \Lambda}\right), \quad \eta \in[0,1], \quad \Lambda \geq 0,
$$

where the $R$ function depends only on the ,Oth moment" (the norm $\left.q_{\infty}\right)$ and the ,,2nd moment" $\left(\left\langle x^{2}\right\rangle_{M}\right)$ of the function $\rho(x)$ restricted to $\Omega_{M}$. Since the moments of the function are independent we can find $\min _{q_{\infty}} R\left(q_{\infty},\left\langle x^{2}\right\rangle_{M}\right)$ keeping $\left\langle x^{2}\right\rangle_{M}$ constant. This step will reduce the state dependent input only to the one quantity $\left\langle x^{2}\right\rangle_{M}$. In order to find the minimum we shall calculate the following derivatives

$$
\frac{\partial}{\partial \eta} R(\eta, \Lambda)=\frac{3}{2}+\frac{1}{2} \ln \left(\frac{(\delta x)^{2} \eta^{3}}{2 \pi e \Lambda}\right), \quad \frac{\partial^{2}}{\partial \eta^{2}} R(\eta, \Lambda)=\frac{3}{2 \eta}
$$

Since for $\eta \in[0,1]$ the second derivative is positive we have only one global minimum $\eta_{\min }=(\sqrt{2 \pi \Lambda} / e \delta x)^{2 / 3}$. On the other hand when $2 \pi \Lambda \geq(e \delta x)^{2}$ the minimum lays outside the domain of $\eta$. Thus, since $R$ is a decreasing function on the interval $\left[0, \eta_{\min }\right]$ we shall modify $\eta_{\min }$ and write

$$
\eta_{\min }(\Lambda)=\min \left\{\left(\frac{\sqrt{2 \pi \Lambda}}{e \delta x}\right)^{2 / 3}, 1\right\} .
$$


Using this result we obtain that:

$$
\begin{cases}R\left(q_{\infty},\left\langle x^{2}\right\rangle_{M}\right) \geq-3\left(\frac{\sqrt{\pi\left\langle x^{2}\right\rangle_{M}}}{2 e \delta x}\right)^{2 / 3} & \text { when }\left\langle x^{2}\right\rangle_{M}<\frac{(e \delta x)^{2}}{2 \pi} \\ R\left(q_{\infty},\left\langle x^{2}\right\rangle_{M}\right) \geq \ln \left(\frac{\delta x}{\sqrt{2 \pi e\left\langle x^{2}\right\rangle_{M}}}\right) & \text { when }\left\langle x^{2}\right\rangle_{M} \geq \frac{(e \delta x)^{2}}{2 \pi}\end{cases}
$$

If we assume that the numbers $M(N)$ are sufficiently large, so that $2 \pi\left\langle x^{2}\right\rangle_{M}<(e \delta x)^{2}$ and $2 \pi\left\langle p^{2}\right\rangle_{N}<$ $(e \delta p)^{2}$ we find the following uncertainty relation

$$
H_{M}^{(x)}+H_{N}^{(p)} \geq-\ln \left(\frac{\delta x \delta p}{e \pi \hbar}\right)-3\left(\frac{\sqrt{\pi\left\langle x^{2}\right\rangle_{M}}}{2 e \delta x}\right)^{2 / 3}-3\left(\frac{\sqrt{\pi\left\langle p^{2}\right\rangle_{N}}}{2 e \delta p}\right)^{2 / 3}>-\ln \left(\frac{\delta x \delta p}{e \pi \hbar}\right)-3
$$

This bound gives a nontrivial limitation when $\delta x \delta p / \hbar \leq \pi e^{-2} \approx 0.425$, what it this case is equivalent to $\left\langle x^{2}\right\rangle_{M}\left\langle p^{2}\right\rangle_{N}<\hbar^{2} / 4$. In a general case the uncertainty relation for the sum of the entropies is $H_{M}^{(x)}+H_{N}^{(p)} \geq \mathcal{L}$, where:

$$
\mathcal{L}=\left\{\begin{array}{ll}
-\ln \left(\frac{\delta x \delta p}{e \pi \hbar}\right)-3\left(\frac{\sqrt{\pi\left\langle x^{2}\right\rangle_{M}}}{2 e \delta x}\right)^{2 / 3}-3\left(\frac{\sqrt{\pi\left\langle p^{2}\right\rangle_{N}}}{2 e \delta p}\right)^{2 / 3} & \text { when }\left\langle x^{2}\right\rangle_{M}<\frac{(e \delta x)^{2}}{2 \pi}, \text { and }\left\langle p^{2}\right\rangle_{N}<\frac{(e \delta p)^{2}}{2 \pi} \\
-3\left(\frac{\sqrt{\pi\left\langle x^{2}\right\rangle_{M}}}{2 e \delta x}\right)^{2 / 3}-\ln \left(\frac{\delta x \sqrt{2\left\langle p^{2}\right\rangle_{N}}}{\sqrt{e \pi} \hbar}\right) & \text { when }\left\langle x^{2}\right\rangle_{M}<\frac{(e \delta x)^{2}}{2 \pi}, \text { and }\left\langle p^{2}\right\rangle_{N} \geq \frac{(e \delta p)^{2}}{2 \pi} \\
-3\left(\frac{\sqrt{\pi\left\langle p^{2}\right\rangle_{N}}}{2 e \delta p}\right)^{2 / 3}-\ln \left(\frac{\delta p \sqrt{2\left\langle x^{2}\right\rangle_{M}}}{\sqrt{e \pi} \hbar}\right) & \text { when }\left\langle x^{2}\right\rangle_{M} \geq \frac{(e \delta x)^{2}}{2 \pi}, \text { and }\left\langle p^{2}\right\rangle_{N}<\frac{(e \delta p)^{2}}{2 \pi} \\
-\ln \left(\frac{2 \sqrt{\left\langle x^{2}\right\rangle_{M}\left\langle p^{2}\right\rangle_{N}}}{\hbar}\right) & \text { when }\left\langle x^{2}\right\rangle_{M} \geq \frac{(e \delta x)^{2}}{2 \pi}, \text { and }\left\langle p^{2}\right\rangle_{N} \geq \frac{(e \delta p)^{2}}{2 \pi}
\end{array} .\right.
$$

\section{Summary}

I have briefly presented a current status in the topic of entropic uncertainty relations for position and momentum variables. I have pointed out ambiguities appearing in the scientific literature. Finally I have investigated the case of finite number of detectors in an approach using discrete Shannon entropies including experimental accuracies and derived nontrivial, state-dependent lower bound generalizing previous results.

\section{Acknowledgments}

I am specially indebted to Iwo Bialynicki-Birula who inspired and supported all my efforts in the topic of entropic uncertainty relations. I would like to thank Stephen P. Walborn, Fabricio Toscano, Luiz Davidovich and all other members of Faculty of Physics in Federal University of Rio de Janeiro (Instituto de Física in Universidade Federal do Rio de Janeiro) for their hospitality after the 12th ICSSUR/Feynfest Conference. This research was partly supported by a grant from the Polish Ministry of Science and Higher Education for the years 2010-2012. 


\section{References}

[1] I. Bialynicki-Birula and J. Mycielski, Commun. Math. Phys. 44, 129 (1975).

[2] D. Deutsch, Phys. Rev. Lett. 50, 631 (1983).

[3] H. Maassen and J.B.M. Uffink, Phys. Rev. Lett.60, 1103 (1988).

[4] M. H. Partovi, Phys. Rev. Lett. 50, 1883 (1983).

[5] I. Bialynicki-Birula, Phys. Lett. 103 A, 253 (1984).

[6] I. Bialynicki-Birula, Phys. Rev. A 74, 052101 (2006).

[7] M. D. Srinivas, Pramana 25, 369 (1985).

[8] I. Bialynicki-Birula and E. Rudnicki, Entropic uncertainty relations in quantum physics, arXiv:1001.4668 (2010).

[9] V. V. Dodonov, J. Opt. B: Quantum Semiclass. Opt. 4, 98 (2002).

[10] B. Coutinho dos Santos, K. Dechoum, and A. Z. Khoury, Phys. Rev. Lett. 103, 230503 (2009).

[11] A. Saboia, F. Toscano, and S. P. Walborn, Phys. Rev. A 83, 032307 (2011).

[12] A. Peres, Quantum Theory: Concepts and Methods. Kluwer, Dordrecht (1995).

[13] D. Chafaï, Gaussian maximum of entropy and reversed log-Sobolev inequality, Séminaire de probabilitiés, Strasbourg 36, 194-200 (2002).

[14] G. Wilk and Z. Włodarczyk, Phys. Rev. A 79, 062108 (2009).

[15] I. Bialynicki-Birula and Ł. Rudnicki, Phys. Rev. A 81, 026101 (2010).

[16] Ł. Rudnicki, Uncertainty related to position and momentum localization of a quantum state, arXiv:1010.3269v1 (2010).

[17] Ł. Rudnicki, S. P. Walborn and F. Toscano, in preparation.

[18] M. Abramowitz and I.A. Stegun, Handbook of Mathematical Functions. Dover, New York, (1964). 